\title{
USO DAS EQUAÇÕES GENERALIZADAS DE PITZER PARA AVALIAÇÃO TERMODINÂMICA DE GASES
}

\author{
Alfredo Muachia ${ }^{1}$ \\ https://orcid.org/0000-0002-3444-9450 \\ Anatalísio Manuel ${ }^{2}$ \\ https://orcid.org/0000-0002-2566-0887 \\ Jane Marques ${ }^{3}$ \\ https://orcid.org/0000-0002-9104-1208 \\ Maria Lemos ${ }^{4}$ \\ https://orcid.org/0000-0003-1309-1522 \\ Antonio A. Chivanga Barros ${ }^{5}$ \\ https://orcid.org/0000-0001-5922-5368
}

\section{RESUMO}

Recebido: 06.02.2020

Aceito: 15.04 .2020

Publicado: 06.07.2020

A equação do tipo Virial foi a base para a proposta da equação generalizada de Pitzer e que consideram as forças de interação molecular do gás, como preponderantes no comportamento mecânico do sistema. A equação do tipo Virial foi baseada na proposição de um polinómio cujo grau leva em consideração o número de moléculas que interagem entre si. A ausência de dados experimentais para a aplicação da equação do tipo Virial resultou na proposta de correlações generalizadas que possibilitam determinar os parâmetros termodinâmicos, a partir das propriedades críticas e reduzidas de cada gás, além do fator acêntrico que relaciona o tamanho das moléculas. Desta forma, este trabalho tem como objectivo avaliar o comportamento do factor de compressibilidade ( $\mathrm{Zi}$ ) de gases, em função da pressão e da temperatura, baseado num estudo numérico realizado a partir de um programa computacional, desenvolvido em linguagem $\mathrm{C}++$, que usa a equação generalizada de Pitzer. Dos resultados obtidos, foi possível observar a similaridade qualitativa do comportamento e a dependência da pressão e da temperatura do sistema com o factor de compressibilidade. Para estas avaliações, concluiu-se que as forças de atração e repulsão moleculares são mais efetivas para sistemas com alta pressão, pois genericamente, para pressões menores que 200 bar, as forças atrativas são mais intensas e acima desta pressão, prevalecem as forças repulsivas, que resultam na liquefação dos gases. Concluiu-se, também, que o comportamento termodinâmico dos gases tem relação direta com o tipo e as características do gás avaliado.

Palavras Chave: Equação de estado, termodinâmica, Pitzer, fator de compressibilidade.

Uso de las ecuaciones generalizadas de pitzer para la Evaluación termodinámica de gases.

\section{RESUMEN}

La ecuación de tipo Virial fue la base para la propuesta de la ecuación generalizada de Pitzer y se consideraron las fuerzas de la interacción molecular del gas, como preponderantes en el comportamiento mecánico del sistema. La ecuación de tipo Virial fue basada en la proposición de un polinomio cuyo grado lleva en consideración el número de moléculas que interactúan entre si. La ausencia de datos experimentales para la aplicación de la ecuación de tipo Virial resultó en la propuesta de correlaciones generalizadas que posibilitan determinar los parámetros termodinámicos, a partir de las propiedades críticas y reducidas de cada gas, además del factor acéntrico que relaciona el tamaño de las moléculas. De esta forma, este trabajo tiene como objetivo evaluar el comportamiento del factor de compresibilidad (Zi) de gases, en función de la presión y de la temperatura, basado en un estudio numérico realizado a partir de un programa computacional, desarrollado en lenguaje $\mathrm{C}++$, que usa la ecuación generalizada de Pitzer. De los resultados

\footnotetext{
Departamento de Engenharias e Tecnologias (DET), Instituto Superior Politécnico de Tecnologias e Ciências (ISPTEC). E-mail: cruzeiromuachia@gmail.com

2 Departamento de Engenharias e Tecnologias (DET), Instituto Superior Politécnico de Tecnologias e Ciências (ISPTEC). E-mail: anatalisio12@gmail.com

3 Departamento de Engenharias e Tecnologias (DET), Instituto Superior Politécnico de Tecnologias e Ciências (ISPTEC). E-mail: jane.marques@isptec.co.ao

4 Departamento de Engenharias e Tecnologias (DET), Instituto Superior Politécnico de Tecnologias e Ciências (ISPTEC). E-mail: maria.lemos@isptec.co.ao

5 Departamento de Engenharias e Tecnologias (DET), Instituto Superior Politécnico de Tecnologias e Ciências (ISPTEC). E-mail: chivanga.barros@isptec.co.ao
} 
obtenidos, fue posible observar la similitud cualitativa del comportamiento y la dependencia de la presión y de la temperatura del sistema con el factor de compresibilidad. Para estas evaluaciones, se concluyó que las fuerzas de atracción y repulsión moleculares son más efectivas para sistemas con alta presión, pues genéricamente, para presiones menores de 200 bar, las fuerzas atractivas son mas intensas y por encima de esta presión, prevalecen las fuerzas repulsivas, que resultan en la licuefacción de los gases. Se concluyó, también, que el comportamiento termodinámico de los gases tiene relación directa con el tipo y las características del gas evaluado.

Palabras clave: Ecuación de estado, termodinámica, Pitzer, factor de compresibilidad.

Use of pitzer generalized equations for thermodynamic gas assessment.

\section{ABSTRACT}

The Virial equation was the basis for generalized Pitzer equation and considered the interaction forces between molecules, as preponderant to know the mechanical behavior of process. The Virial equation was based on polynomial equation whose degree takes into account the number of molecules that interact with each other. The absence of experimental data for the application of the Virial equation resulted in the proposal of generalized correlations that make it possible to determine the thermodynamic parameters, based on the critical and reduced properties of each gas, in addition to the acentric factor that relates the size of the molecules. For this correlation, was insited the acentric factor that relates with moleculares size. In this way, here were evaluate the behavior of gas compressibility factor (Zi) as function of pressure and temperature, based on a numerical study carried out from a computer program, developed in $\mathrm{C}++$ language, using generalized Pitzer`s equation, for three differents gases. From the results obtained, it was possible to observe the similarity of $\mathrm{Zi}$ behavour and its dependence with pressure and temperature. For this, we concluded that molecular attraction and repulsion forces are more effective for systems with high pressure. The attractive forces are more intense above 200 bar, when the repulsive forces prevail. It was also concluded that thermodynamic gases behavior is directly related to characteristics of type of gas evaluate.

Key words: State Equations, Thermodinamic, Pitzer Equation, Compressibility Factor.

\section{Introdução}

A resolução de problemas de Engenharia Química exige a predição de propriedades de substância constituintes das fases líquida e vapor, tanto como componentes puros bem como para misturas. Para a avaliação da fase vapor/gasosa, é comum utilizar as equações de estado de gás ideal, cúbicas, tipo Virial ou as generalizadas. Todas as equações referenciadas relacionam o fator de compressibilidade (Z) da substância pura com os parâmetros macroscópicos e mensuráveis, tais como Pressão, Volume e Temperatura, geralmente descritas como PVT. A relação, assim referenciada, possibilita avaliar o comportamento termodinâmico dos sistemas pois, existem mecanismos para a determinação experimental destes parâmetros que garantem o conhecimento de diversas relações físicas, associadas a possível perturbação de cada uma delas. Pode-se destacar que os sistemas pressurizados, caracterizados pelo aumento da pressão promovem a diminuição do volume do sistema, tendo como consequência, forte interação molecular, o que resulta no incremento das forças de atração e repulsão das moléculas constituintes do sistema em análise.

A análise destes sistemas deve ser fundamentada, preliminarmente, pelo conhecimento do comportamento de um gás ideal, um gás hipotético formado por moléculas e caracterizado pela ausência das forças intermoleculares e cujos choques são inexistentes ou desprezíveis e os fenómenos são todos baseados nos princípios de conservação do momento e da energia cinética (Peter Atkins, J. e De Paula, 2012)..

Por outro lado, quando da avaliação do comportamento dos gases reais, observa-se um forte desvio do comportamento ideal, por se considerar que, para sistemas sustentados por estes princípios, com o aumento da pressão, ocorre uma forte aproximação entre as moléculas do sistema, potencializando-se as forças de interação molecular, característicos dos fenómenos de atração e repulsão entre elas. Para estes casos, o factor de compressibilidade é incluído na equação, para representar as forças referenciadas (Van Wylen et al, 2003).

\section{CONCEPÇÃO TEÓRICA}

Para Rowland and May (2015), o estado de um gás puro é especificado pelo seu volume, V, número de moles, $\mathrm{n}$, pressão, $\mathrm{P}$, temperatura, $\mathrm{T}$, do sistema e três destas variáveis são independentes e sustentam a descrição das equações de estado, geralmente representado conforme a Equação (1). 


$$
P=f(T, V, n)
$$

A aplicação da Equação (1) para sistemas cuja pressão tende a zero, possibilita a definição da equação de estado de gás ideal, conforme a Equação (2).

$$
P V=n R T
$$

A Equação (2) é aplicada para sistemas com pressão tendente a zero, caracterizados por um forte distanciamento entre as moléculas constituintes e portanto, pela ausência das forças de interação moleculares. Existem trabalhos na literatura que confirmam os princípios dos fenómenos associados à Equação (2), principalmente para gases ideais. Contudo, estudos de incremento da pressão dos sistemas têm sido realizados para melhor compreensão dos efeitos das forças moleculares em sistemas pressurizados, que envolvem mudança de fase ou sistemas caracterizados por operarem em condições supercríticas. O incremento das pressões resulta no aumento das forças de interação entre as moléculas, tendo como principais fenómenos a observação das forças de atração e repulsão que afetam direta ou indiretamente o volume total do gás e a pressão do sistema, principalmente para processos isotérmicos (Adacid et al, 1988). A necessidade de compreensão dos fenómenos referenciados resultou na proposição de equações de estado para gases reais ou não ideais. Entre as diversas equações termodinâmicas, destaca-se a equação do tipo Virial, que introduz uma série polinomial, caracterizada pela Equação (3).

$$
P V=R T\left(1+B^{\prime} P+C^{\prime} P^{2}+\cdots \ldots=R T\left(1+\frac{\mathrm{B}}{\mathrm{V}}+\frac{\mathrm{C}}{V^{2}}\right) \ldots \cdots \cdots\right.
$$

Onde B, B', C, C' . . . são o segundo, terceiro, ... coeficientes de virial e que dependem da temperatura. O primeiro coeficiente de viral é igual a um (1) e a temperatura de Boyle é aquela no qual o coeficiente $\mathrm{B}$ se anula. Como os demais termos têm um valor, geralmente, reduzido, o gás comporta-se de forma perfeita num intervalo maior de pressão. Como para pressões baixas, B é o único fator importante, desta forma, pode-se observar que a derivada do fator de compressibilidade em função da pressão, para a pressão tendente a zero, é igual a zero (Equação 4).

$$
\frac{\partial \mathrm{z}}{\partial \mathrm{p}}=0 \operatorname{para} P \rightarrow 0
$$

O gás, ao ser comprimido, de acordo com Simoes et al (2016), a temperatura constante, ocorre eventualmente o fenómeno de condensação. A pressão de vapor ou de saturação é aquela que resulta na coexistência do líquido e do vapor em equilíbrio entre si e que pode se estender por uma gama de volumes molares, que diminui com o aumento da temperatura, formando uma linha horizontal num diagrama Pressão x Volume (PV). A partir de uma dada temperatura, este patamar converge num ponto crítico. Os volumes, pressões e temperaturas correspondentes são definidos como críticos.

Por isto, para o estudo do comportamento dos gases, dada a complexidade referenciada, foram propostas diversas equações de estado, especialmente às cúbicas, destacando-se a de Van Der Walls, Redlich-Kwong, Soave-Redlich-Kwong, entre outras. Entretanto, foram as equações generalizadas desenvolvidas a partir da equação do tipo virial que têm sido mais utilizadas no estudo do comportamento de gases, devido as diversas correlações propostas para o cálculo dos coeficientes do virial associados (Simoes et al, 2016).

Entre as diversas correlações, destaca-se aquela proposta por Pitzer que expressa o fator de compressibilidade, $\mathrm{Zi}$, como uma função de temperatura reduzida, $\mathrm{Tr}$, pressão reduzida, $\mathrm{Pr}$, e do fator acêntrico w do componente. 
A correlação generalizada de Pitzer é um modelo semi-empírico que, quando aplicada possibilita a compreensão do comportamento das forças de interação molecular, principalmente quando envolve soluções que resultam na acção de forças entre moléculas com tamanhos diferentes. Esta correlação foi descrita pela primeira vez pelo químico Kenneth Pitzer (Simoes et al, 2016) e caracteriza as interações entre moléculas ou ions, quando em solução, através de combinações lineares de parâmetros de uma expansão do tipo virial, da energia livre de Gibbs em excesso.

Além disso, a correlação de Pitzer é muito eficiente na previsão do comportamento das soluções eletrolíticas que abrangem soluções infinitamente diluídas até concentradas. Por outro lado, o modelo de Pitzer é na sua essência empírico e os parâmetros de virial que representam as interações de curto alcance não podem ser diretamente correlacionados com as propriedades da solução e, portanto, não podem ser extrapoladas para diferentes casos (Rowland and May (2015), Simoes et al (2016) e May and Rowland (2017)).

As equações generalizadas são usadas em muitas aplicações, pois abrangem correlações específicas para o segundo, terceiro e quarto coeficiente do tipo virial. A extensão polinomial da equação aumenta a sua abrangência e por isto, se comparadas, a do terceiro coeficiente fornece resultados mais precisos em relação com aqueles oriundos do segundo coeficiente (Rowland and May (2015). O comportamento PVT predito por uma equação de estado cúbica, em certas condições e para certos gases, não é tão preciso quanto aos resultados oriundos das equações generalizadas. Por outro lado, para descrever, com maior precisão, o comportamento PVT de fluídos, numa faixa ampla de temperatura e pressão, requer-se uma equação de estado mais completa do que a equação tipo virial em função da ausência de dados experimentais necessários para a determinação dos respectivos coeficientes.

Por isto, a generalização de equações possibilita a análise mais profícua do comportamento dos gases, pois esta não é complexa e é de fácil solução numérica e analítica. Estas equações são baseadas nas correlações que levam em considerações as propriedades críticas dos gases e são denominadas de Equações de Estado Generalizadas (EEG) e podem ser aplicadas para diversos tipos de gases (Adacid et al. , 1988).

Para Bertoli et. al. (2017), as equações de estado são de grande importância no estudo da termodinâmica pois, são usadas para correlacionar um certo número de parâmetros presentes na avaliação de processos de Engenharia Química e que possibilitam o cálculo das propriedades de um gás em diversas condições, destacando-se o fator de compressibilidade $\left(Z_{\mathrm{i}}\right)$, parâmetro que leva em consideração as forças de interação entre as moléculas constituintes da mistura.

O fator de compressibilidade do componente $\left(Z_{\mathrm{i}}\right)$ é uma variável que depende diretamente da pressão e do volume do sistema e é inversamente proporcional a temperatura e ao número de moles dos componentes constituintes da mistura em estudo, cuja expressão matemática é representada pela Equação (5).

$$
Z_{i}=\frac{\mathrm{PV}}{\mathrm{n}_{i} \mathrm{RT}}(5)
$$

A aplicação e a análise dos resultados oriundos da Equação (5) mostram que, para os gases perfeitos ou ideais o fator de compressibilidade é igual a 1. Os gases ideias são caracterizados pela ausência de forças de interação, e, portanto, forças de repulsão e atração, entre as moléculas que compõem o sistema gasoso. Para os gases não ideais, caracterizados pela forte interação entre as moléculas, o fator de compressibilidade mede o grau de não idealidade e, geralmente, quando o fator de compressibilidade é calculado este é diferente de um (1) e seus valores dependem do afastamento do comportamento do gás perfeito ou ideal. Para tais gases, os fenómenos associados relacionam-se com a predominância das forças repulsivas $\left(Z_{i}>1\right)$ ou forças atrativas $\left(Z_{i}<1\right)$, de acordo com a descrição da Figura 1 (Adacid et al (1988), Russel (2009), Rowland and May (2015) e Simoes et al, (2016)). 
Figura 1: Factor de Compressibilidade como função da pressão, a T constante.

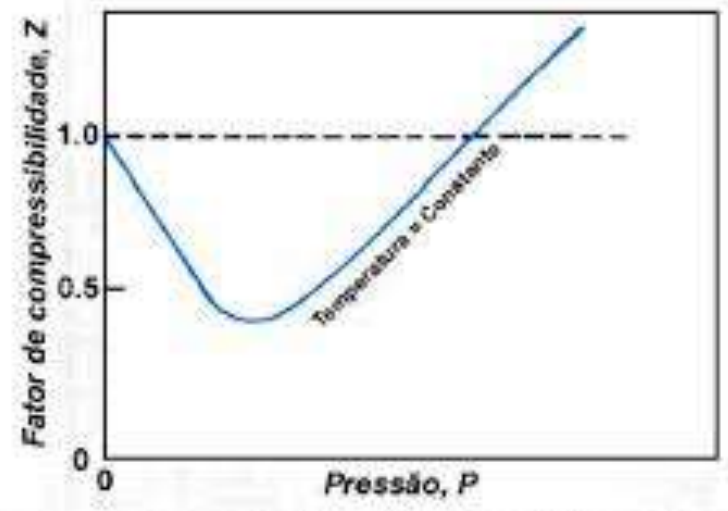

Fonte: Beggs (1985)

O fator de compressibilidade é um parâmetro de correção que descreve os fenómenos físicos associados aos gases ideais e reais, baseados nas forças atuantes entre as moléculas constituintes de uma dada mistura de gases. Para Russel (2009), o desvio do comportamento do gás ideal é mais evidente quando as pressões do sistema são altas e baixas temperaturas. Para sistemas conservados em altas pressões, o fator de compressibilidade de um gás real pode ser maior ou menor que 1,0 . Este comportamento pode ser identificado no diagrama de compressibilidade de substâncias puras (Figura 1), na curva de gás real. Na sua generalidade, as características desta curva se semelham ao comportamento de quase todos os gases reais, principalmente do ponto de vista qualitativo, em função da pressão do sistema. Por outro lado, este comportamento depende, quantitativamente, da temperatura e das pressões do sistema e das propriedades críticas de cada substância avaliada. Os estudos apresentados neste artigo foram sustentados pela ausência de análise mais profícua do comportamento do fator de compressibilidade, de diferentes tipos de gases, através do uso e análise das equações generalizadas de Pitzer, truncadas no segundo e terceiro termos, respectivamente. Desta forma, a equação do fator de compressibilidade baseado no $2^{\circ}$ coeficiente do tipo virial está presente na Equação 6 e do $3^{\circ}$ coeficiente do tipo virial na Equação (7).

$$
\begin{gathered}
Z_{i}=1+B_{i}^{o} \frac{P_{r i}}{T_{r i}}+w_{i} B_{i}^{1} \frac{P_{r i}}{T_{r i}} \\
(6) \\
Z_{i}=1+\left(B_{i}^{o}+w_{i} B_{i}^{1}\right) \frac{P_{r i}}{T_{r i}}+\left(C_{i}^{o}+w_{i} C_{i}^{1}\right) \frac{P r^{2}}{T r^{2}}
\end{gathered}
$$

Onde $P_{r i}=\frac{P}{P_{C i}}$ e $T_{r i}=\frac{T}{T_{r i}}$ são as pressões e temperaturas reduzidas do gás.

A equação de Pitzer é uma equação de fácil implementação numérica e analítica e é obtida baseada nas correlações que dependem das propriedades críticas dos gases constituintes da mistura. Embora a equação generalizada seja sustentada em dois parâmetros termodinâmicos (Tri e Pri), a sua implementação garante resultados mais precisos, se comparados com aqueles advindos da equação do gás ideal ou equações de estado cúbicos, pois a avaliação é baseada no segundo ou terceiro coeficientes da equação do tipo Virial (Equação 6 ou 7) que levam em consideração a possibilidade de interação entre duas ou três moléculas, simultaneamente. Por outro lado, a equação generalizada incorpora um parâmetro característico da estrutura molecular do gás, fator acêntrico $\left(w_{i}\right)$ com uma forte relação com a pressão e a temperatura do sistema, através do tamanho das moléculas constituintes da mistura em estudo (Russel, 2009). Para isto, foram 
propostas correlações apropriadas, todas funções da pressão reduzida, conforme as Equações 8, 9 e 10 (Smith, Ness Van e Abbott, 2007).

$$
\begin{gathered}
B_{i}^{o}=0,083-\frac{0,422}{T_{i}^{1,6}}(8) \\
B_{i}^{1}=0,139-\frac{0,172}{T_{i}^{4,2}}(9) \\
C_{i}^{o}=0,01407+\frac{0,02432}{T_{r}}-\frac{0,00313}{T_{i}^{10,5}} \\
C_{i}^{1}=-0,02676+\frac{0,05539}{T_{i}^{2,7}}-\frac{0,00242}{T_{i}^{10,5}}
\end{gathered}
$$

As Equações 8, 9, 10 e 11 possibilitam o cálculo dos coeficientes de interação dos componentes, utilizados nas Equações 6 e 7 que resultam na determinação do fator de compressibilidade, tanto quanto da interação entre duas ou entre três moléculas constituintes da mistura em estudo.

\section{Metodologia}

Para este trabalho, foi desenvolvido um algoritmo para o cálculo do factor de compressibilidade, utilizando as equações do tipo virial, truncadas no $2^{\circ}$ ou $3^{\circ}$ termo, respectivamente. Com o algoritmo desenvolvido, foi implementado um programa computacional, em linguagem $\mathrm{C}++$, com uma base de dados que contempla especialmente as propriedades críticas dos gases avaliados neste trabalho.

Para este estudo, foram selecionados três tipos de gases, sendo um hidrocarboneto, etileno $\left(\mathrm{C}_{2} \mathrm{H}_{4}\right)$, e dois gases inorgânicos, dióxido de carbono $\left(\mathrm{CO}_{2}\right)$ e monóxido de nitrogénio $(\mathrm{NO})$. A escolha destes gases deve-se a grande diferença nas suas estruturas moleculares e as características das respectivas ligações químicas, que devem resultar em comportamentos termodinâmicos diferentes, tanto no âmbio deste estudo como na aplicação em processos da indústria química.

Os gases selecionados e o algoritmo desenvolvido possibilitaram avaliar o comportamento do fator de compressibilidade, em função da pressão e da temperatura do sistema. Para isto, explorou-se uma faixa ampla de pressão, para diferentes temperaturas de operação e avaliado o comportamento deste parâmetro termodinâmico. Os resultados obtidos foram avaliados e permitiram estabelecer uma comparação do comportamento dos gases estudados para as duas equações analisadas.

\section{Resultados e discussões}

A análise do fator de compressibilidade, a partir da equação generalizada de Pitzer, com o segundo e o terceiro coeficientes, resultou na descrição gráfica da Figura 2. A equação generalizada de Pitzer truncada no segundo termo, mostra o comportamento linear do factor de compressibilidade para os três gases estudados.

Para este caso, (Figura 2a), o fator de compressibilidade diminui com o aumento da pressão, para o etileno e dióxido de carbono, ocorrendo comportamento inverso para o monóxido de nitrogénio. O comportamento referenciado mostra que, nas mesmas condições de operação, as forças resultantes da pressão do sistema agem de formas diferentes, caracterizadas por um forte efeito da repulsão quando o monóxido de oxigênio é avaliado e atração quando o etileno e dióxido de carbono são avaliados. Este comportamento é acentuado com o aumento da pressão do sistema. Os desvios observados foram baseados no afastamento do comportamento de um gás ideal, cujas características foram definidas neste trabalho.

A análise baseada na equação generalizada com o terceiro coeficiente tipo virial (Figura 2b) mostra o comportamento parabólico do fator de compressibilidade em função da pressão. Para este caso, é possível observar a ação das forças de atração e repulsão predominantes para faixas diferentes de pressão, para o mesmo gás e, por isto, de melhor representação do comportamento fenomenológico descrito na literatura (Adacid et al (1988), Russel (2009), Rowland and May (2015) e Simoes et al, (2016)), caracterizado pela coexistência de regiões com fator de compressibilidade menor que um e maiores que um para a segunda faixa de pressão. Nesta figura, o monóxido de 
nitrogênio comporta-se inicialmente como gás ideal até pressões de aproximadamente 200 bar e em seguida, passam a prevalecer as forças de repulsão que resultam num fator de compressibilidade maior que um. Para o etileno e dióxido de carbono, observa-se efeitos das forças de atração até 400 bar, aproximadamente, e em seguida a predominância das forças repulsivas, com maior incidência sobre o gás orgânico.

Figura 2. Comportamento do Z em função da P para T de 498,15K (a: $2^{\circ}$ coeficiente e b: $3^{\circ}$ coeficiente)

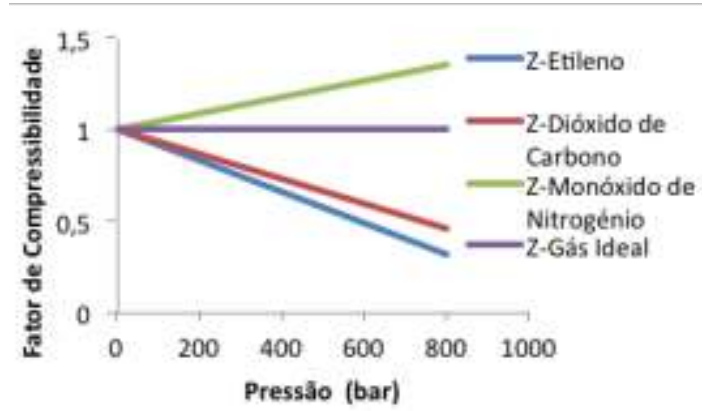

(a)

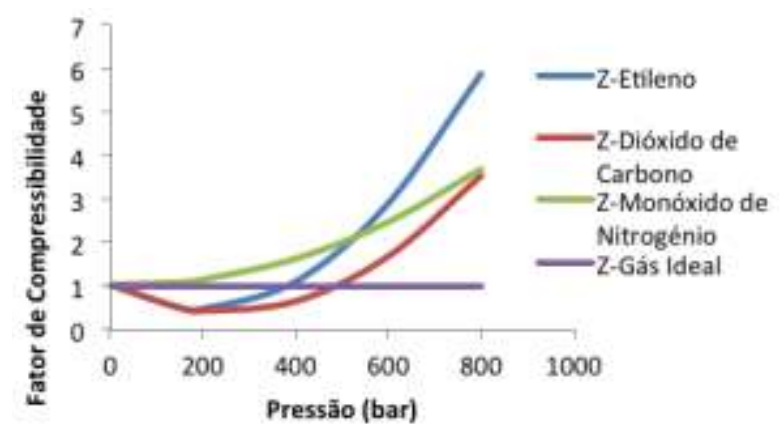

(b)

A análise comparativa do comportamento de um dado gás, utilizando-se as equações truncadas no segundo e terceiro termos é apresentada na Figura 3 que descreve a influência do terceiro coeficiente na equação sobre o comportamento do fator de compressibilidade dos gases avaliados. Para este caso, dependendo do grau do polinómio utilizado, o comportamento fenomenológico associado as forças de repulsão e atração é adverso, com prevalência das forças de atração para a equação com o $2^{\circ}$ termo e repulsão para a equação do $3^{\circ}$ termo. Este comportamento é similar para os dois gases estudados e representados na Figura 3 (a e b) o que exige a exploração de outros modelos de equações para melhor representarem o comportamento progressivo da ação da pressão, sobre o comportamento do processo.

Figura 3. Comportamento do Z em função da P para T de 498,15K (a: etileno e b: Dióxido de carbono)
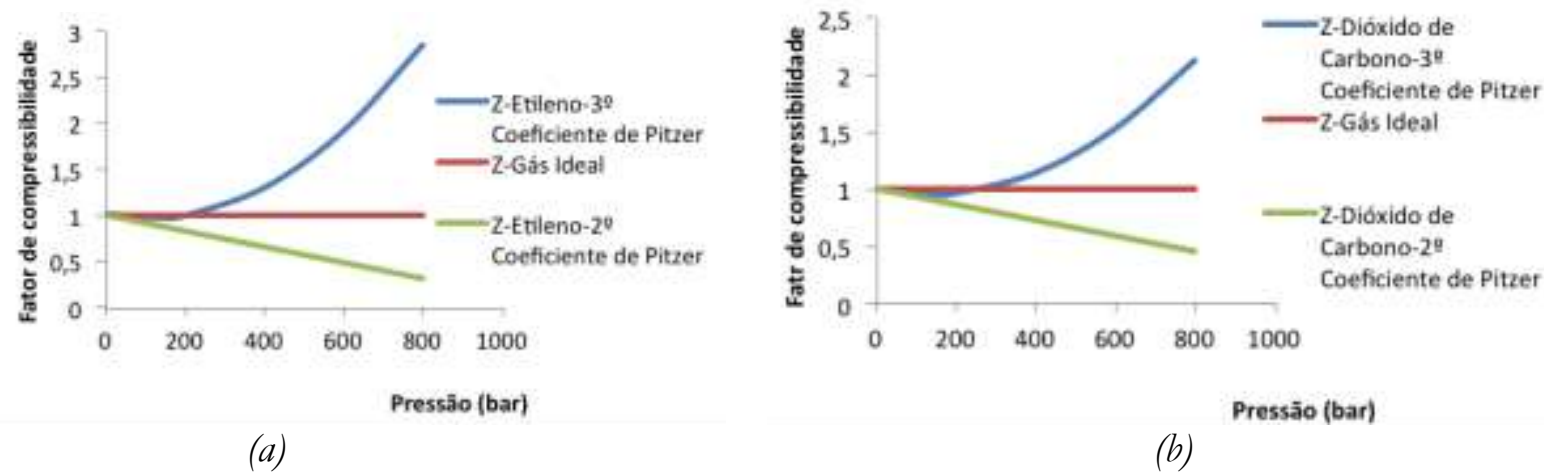

$\mathrm{Na}$ análise da Figura 3, ressalta-se que quando a avaliação do comportamento dos gases é realizada a baixas pressões, o comportamento se assemelha aquele dos gases ideais, caracterizados por um fator de compressibilidade de aproximadamente igual a um (1), em função das grandes distâncias entre as moléculas presentes no sistema, decorrente de grandes volumes do gás e reduzida massa destes, contida no sistema. 
Fez-se a análise da influência da temperatura sobre o comportamento do fator de compressibilidade, em função da pressão do sistema, tendo-se observado que para temperaturas reduzidas, os efeitos das forças de atração e repulsão prevalecem explicitamente, com a observação de duas regiões distintas (Figura 4), para os dois gases avaliados. Entretanto, com o aumento da temperatura dos gases e consequente aumento da energia interna do sistema, predominam as forças repulsivas para altas pressões, mas para pressões até aproximadamente 350 bar, o fator de compressibilidade se aproxima daquele característico do gás ideal.

Figura 4. Comportamento do Z em função da P para três temperaturas diferentes (a: Dióxido de carbono e b: etileno)

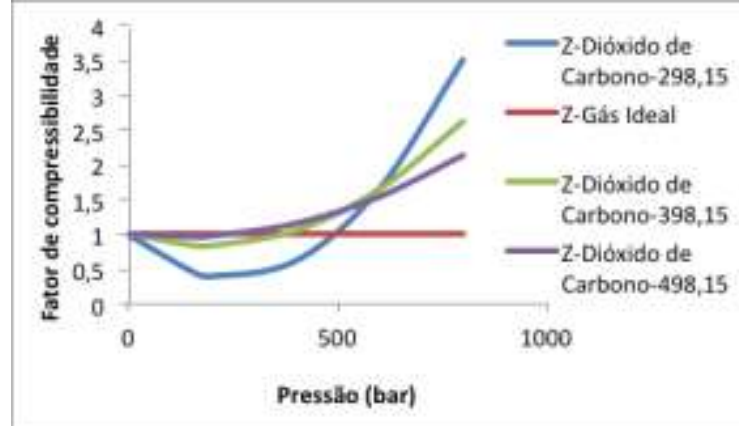

(a)

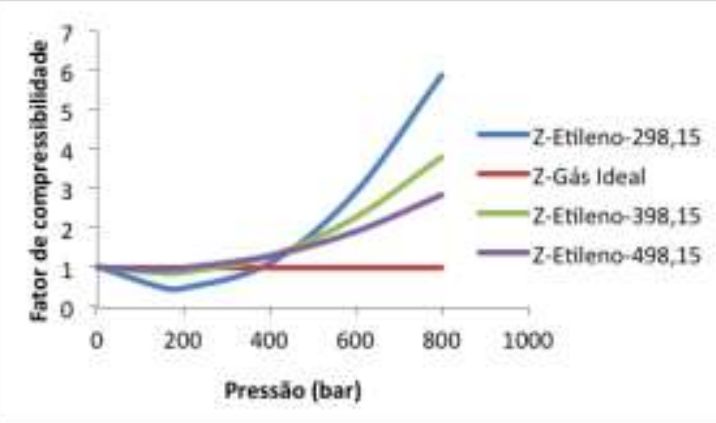

(b)

\section{Conclusões}

Os resultados aqui apresentados mostraram a validade da metodologia adotada neste estudo, com realce para o alcance dos objectivos previamente estabelecidos. Contudo, a análise dos dados contidos neste artigo mostra a necessidade de se aprofundar estudos termodinâmicos para melhor avaliação do comportamento dos gases, explorando-se diversas equações de estado existentes e propor outras mais representativas e capazes de compor o comportamento real dos processos. Contudo, o entendimento dos fenómenos para gases puros possibilita estender as análises para misturas de gases com tamanhos de moléculas diferentes. Neste contexto, foi possível concluir que:

i. O fator de compressibilidade dos gases apresenta forte dependência com a pressão do sistema e geralmente o seu comportamento é dependente do gás utilizado;

ii. A dependência do fator de compressibilidade com a pressão depende do termo de truncamento da equação, pois para equação truncada no segundo termo, o comportamento do Z é linear e para o truncamento no terceiro termo, o comportamento é parabólico;

iii. A temperatura do gás afeta diretamente o comportamento do fator de compressibilidade. Geralmente, quanto maior a temperatura, maiores as forças de atração e repulsão, nas faixas de pressão muito bem definidas. Altas temperaturas prevalecem as forças de repulsão que resultam em maiores volumes do sistema;

iv. O estudo aqui apresentado possibilita maior compreensão dos fenómenos associados à relação PVT dos gases, comumente presentes nos processos da indústria química e petroquímica.

\section{Referências bibliograficas}

Adacid, Yoshinori; Fijihara, Ichiro; Takamiya, Masaaki and Nakanishi, Koichiro (1988). Generalized equation of state for Lennard-Jones fluids-I. Pure fluids and simple mixtures.

Fluid Phase Equilibria Journal. Volume 39. United State of America. (Pp. 1-38). DOI: www.doi.org/10.1016/0378-3812(88)80001-3.

Atkins, Peter e De Paula, Júlio (2012). Físico-Química. 9ª ed., Vol 1; Editora LTC. Brasil. 
Beggs, H. Dale (1985). Gas Production Operations. Editora: Indra Bayu. United State of America.

Bertoli, Sávio L.; Kalvelage, Pollyana M. S., Albuquerque, Allan A. and Barros, António A. Chivanga (2017). (Vapor + Liquid) Equilibrium for Mixtures Ethanol + Biodiesel from Soybean Oil and Frying Oil; International Journal of Thermodynamics. Vol. 20. Turquia. (Pp. 159-164).

May, Peter and Rowland, Darren (2017). Thermodynamic Modeling of Aqueous Electrolyte Systems: Current Status. J. Chem. Eng. Data. 62, 9, United State of America (Pp. 24812495). DOI: www.doi.org/10.1021/acs.jced.6b01055.

Rosenberg, Yoav Oved; Metz, Volker and Ganor, Jiwchar (2011). Co-Precipitation of Radium in High Ionic Strength Systems: 1. Thermodynamic Properties of the Na-Ra-Cl-SO $\mathrm{S}_{4}-\mathrm{H}_{2} \mathrm{O}$ System - Estimating Pitzer Parameters for $\mathrm{RaCl}_{2}$. Cosmochimica Acta Journal. Volume 75, Issue 19. Pages 5367-5818. doi: 10.1016/j.gca.2011.06.042. Alemanha.

Rowland, Darren and May, Peter M. (2015). Comparison of the Pitzer and Hückel Equation Frameworks for Activity Coefficients, Osmotic Coefficients, and Apparent Molar Relative Enthalpies, Heat Capacities, and Volumes of Binary Aqueous Strong Electrolyte Solutions at $25^{\circ}$ C. J. Chem. Eng. Data. www.doi.org/10.1021/acs.jced.5b00161. United State of America.

Russel, John B. (2009); Química Geral. Volume I, 2a Edição. Editora Mkron Bokks. Brasil.

Simoes, Marcus C.; Hughes, Kevin J.; Ingham, Derek B.; Ma, Lin and Pourkashanian, Mohamed (2016). Estimation of the Pitzer Parameters for 1-1, 2-1, 3-1, 4-1, and 2-2 Single Electrolytes at $25^{\circ} \mathrm{C}$. J. Chem. Eng. Data. 61, 7. United States of America (Pp. 2536-2554). DOI: www.doi.org/10.1021/acs.jced.6b00236.

Smith, Ness Van e Abbott, Hendrick C. (2007). Introdução à Termodinâmica da Engenharia Química. $7^{a}$ edição. Editora LTC. Brasil.

Van Wylen, Gordon J.; Sonntag, Richard E. e Borgnakke, Sontag Claus (2003). Fundamentos da Termodinâmica Clássica. Tradução da $4^{\mathrm{a}}$ Edição. Editora Edgard Blücher. Brasil. 